\title{
Differences in the magnetic resonance imaging parameter T2* may be identified during the course of canine patellar tendon healing: a pilot study
}

\author{
Sarah L. Pownder ${ }^{1} \wedge$, Kei Hayashi ${ }^{2}$, Bin Q. Lin ${ }^{1}$, Kathleen N. Meyers ${ }^{1}$, Brian G. Caserto ${ }^{3}$, Ryan E. Breighner ${ }^{1 \wedge}$, \\ Hollis G. Potter ${ }^{1}$, Matthew F. Koff ${ }^{1 \wedge}$ \\ ${ }^{1}$ MRI Laboratory, Hospital for Special Surgery, New York, NY, USA; ${ }^{2}$ Cornell University College of Veterinary Medicine, Ithaca, NY, USA; \\ ${ }^{3}$ VetPath Services, Stone Ridge, NY, USA \\ Correspondence to: Matthew F. Koff, PhD. MRI Laboratory, Hospital for Special Surgery, 535 East 70th Street, New York, NY 10012, USA. \\ Email: koffm@hss.edu.
}

\begin{abstract}
Background: Previous studies have utilized ultrashort echo (UTE) magnetic resonance imaging (MRI), and derived $\mathrm{T} 2 *$ maps, to evaluate structures with highly ordered collagen structures such as tendon. T2* maps may provide a noninvasive means to assess tendon damage and healing. This pilot study evaluated the longitudinal relationship of an induced mechanical strain on the patellar tendon with corresponding UTE T2* metrics, histologic and biomechanical evaluation at two post-operative time points.

Methods: A total of 27 patellar tendons in male Beagles were surgically subjected to stretching by a small diameter $(\mathrm{SmD})$ or a large diameter $(\mathrm{LgD})$ diameter rod to induce damage due to strain, and evaluated at 4and 8-week intervals using quantitative MRI (qMRI), biomechanical testing, and histology. A separate set of 16 limbs were used as controls.

Results: The tendons experienced a $67 \%$ and $17 \%$ prolongation of short $\mathrm{T} 2 *$ values as compared to controls at 4 and 8 weeks post-operatively, respectively. Histologic analysis displayed a trend of increased collagen disruption at 4 weeks followed by presence of greater organization at 8 weeks. Biomechanical evaluation found a reduction of tendon modulus and failure strain at both time points, and an increase in cross-sectional area at 4 weeks as compared to controls.

Conclusions: These findings display tendon healing in response to an imposed strain and present the utility of qMRI to evaluate longitudinal differences of patellar tendon T2* values in a model of induced subclinical tendon damage. The qMRI technique of UTE provides a means to non-invasively evaluate the healing process of a mechanically damaged tendon.
\end{abstract}

Keywords: Magnetic resonance imaging (MRI); tendon; ultrashort echo (UTE); T2*

Submitted May 21, 2020. Accepted for publication Oct 28, 2020.

doi: 10.21037/qims-20-684

View this article at: http://dx.doi.org/10.21037/qims-20-684

\section{Introduction}

Patellar tendinopathy, also known as "jumper's knee" (1), manifests clinically as a combination of diffuse or localized swelling, pain and/or reduced functional performance (2), and exhibits disorganization of collagen fibers histologically $(3,4)$. Patellar tendinopathy is commonly seen in basketball, volleyball, football and soccer athletes $(1,5,6)$ and affects $25 \%$ to $50 \%$ of athletes, with a disproportionate number

^ ORCID: Sarah L. Pownder, 0000-0003-2692-7384; Ryan E. Breighner, 0000-0002-2190-3597; Matthew F. Koff, 0000-0001-6969-0183. 
being basketball players (5). The distinct cause of patellar tendinopathy is unclear but is related to the load bearing capability of the patellar tendon to excessive forces from the quadriceps muscle, providing movements that require rapid knee flexion and acceleration and deceleration, and high frequency repetitive movements $(7,8)$.

Animal models may provide a means to evaluate the progression and resolution of patellar tendinopathy. Models of patellar tendinopathy have been developed in mice $(9,10)$, rats $(11,12)$, rabbits (13), and pigs (14). These models include induction of damage by surgical excision or punch excision of portions of tendon (9), chemical administration (11), or the use of loading devices (12). Each species model has intrinsic advantages and disadvantages relating to lesion generation/surgical intervention, postoperative care and rehabilitation protocols, non-invasive imaging, and postmortem testing. While rodent models are commonly used, the ease afforded by the larger anatomy in non-rodent models is attractive as this permits the use of surgical instruments and clinical imaging equipment that is available and approved for use in humans. Canine models of cruciate ligament reconstruction have been developed using the patellar tendon as a graft $(15,16)$ but to the best of our knowledge, a canine model of patellar tendinopathy has not been developed. Since the patellar tendon is infrequently injured in dogs $(17,18)$ a canine model of patellar tendinopathy would minimize the chance for clinically silent pathology in this species to confound study outcomes. The dog is also ideal from a standpoint of behavior and interaction. The domestic dog can be trained to run on a treadmill, sit and stand with weights, and walk over a force plate for objective gait analysis (19). In addition, size of the canine easily permits use of standard clinical imaging equipment for post-operative monitoring of tendon healing in lieu of direct histologic processing.

Magnetic resonance imaging (MRI) has shown the greatest utility for evaluation of tendon injury (18) in the dog. MRI is an optimal non-invasive imaging modality for evaluating tendons in vivo due to its multi-planar imaging capabilities and superior soft tissue contrast. However, tendons typically exhibit little signal on standard clinical fast-spin echo (FSE) sequences due to their highly ordered collagen composition and corresponding short transverse relaxation times (T2). Only when a tendon is highly disrupted, inflamed or edematous do abnormalities manifest on standard FSE images while subtle injuries may escape detection (20-22). In addition, morphologic image interpretation relies on subjective assessment of signal intensity (SI), which does not correlate to any specific histologic characteristic. Newer quantitative MRI (qMRI) techniques, such as ultrashort echo (UTE) time imaging, can visualize tissue species with very short $\mathrm{T} 2$ values ( $\mathrm{T} 2 \leq 5 \mathrm{~ms}$ ), permitting calculation of $\mathrm{T} 2$, a reproducible decay constant (21). Prolongation of T2* may be used as a biomarker of disruption in highly ordered tissues such as tendons, ligaments, menisci, and periosteum $(20,23,24)$, detecting early changes of structural damage (25-27). In addition, while T2 values of articular cartilage have been correlated to its mechanical properties (28-30), similar studies have not been performed for tendon T2* values. Relating these metrics to mechanical and histologic correlates of damaged and repaired tendons will facilitate integration of these techniques into clinical care for deciding an appropriate course of treatment and postoperative evaluation.

Therefore, the purpose of this pilot study was to induce mechanical damage to the patellar tendon and evaluate temporal differences of UTE T2* values in comparison to histologic and biomechanical outcomes. We hypothesized that prolongation due to a controlled initial damage event, followed by shortening of $\mathrm{T} 2{ }^{*}$ could be used as a biomarker of disruption and healing of the patellar tendon, respectively.

\section{Methods}

\section{Strain validation}

Cadaveric and physical models were used to verify the magnitude of applied strain to be used in the in-vivo portion of the study. Cadaveric canine pelvic limbs were fixed to wooden blocks. Next, latex bands with length dimensions similar to the canine patellar tendon were also secured to four wooden blocks. Nylon suture (7-0) was used to secure 11 to 16 metal beads of $2 \mathrm{~mm}$ diameter along the length of the tendon and latex bands. Bead placement was performed carefully to prevent local puckering of the tendon or latex band. Computer tomography (CT) positional markers were adhered to each wood block to account for specimen/model positional differences between scanning series. CT of the intact limbs and model were acquired on a GE Discovery CT750 HD scanner (GE Healthcare, Waukesha, WI, USA) with the parameters: $120 \mathrm{kVp}, 250 \mathrm{~mA}, 0.312 \mathrm{~mm}$ slice interval, $0.625 \mathrm{~mm}$ slice thickness, $0.248 \mathrm{~mm}$ in-plane resolution (127 mm FOV, 512 matrix). A bone convolution kernel (edge-enhancing) was used. Next, a metal rod of 


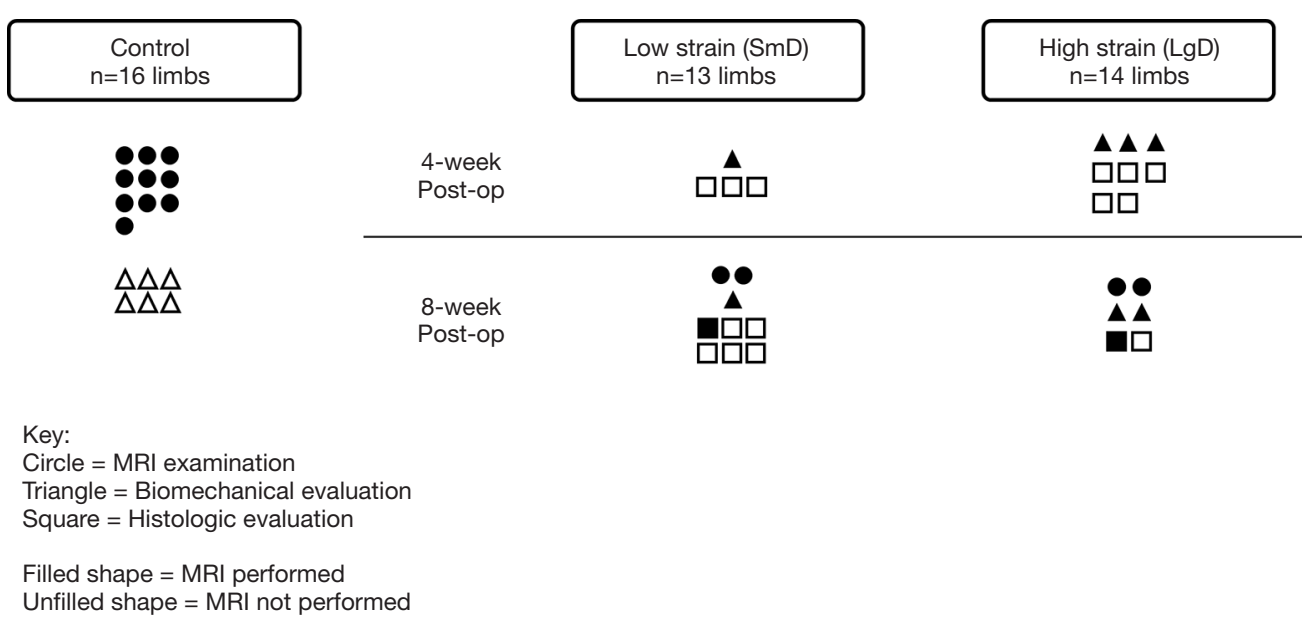

Figure 1 Chart displaying the number of limbs within each group and the evaluations performed at each time point over the course of the study. Each shape displayed represents 1 limb. SmD, small diameter rod used to induce strain; $\mathrm{LgD}$, large diameter rod used to induce strain; MRI, magnetic resonance imaging.

$4.76 \mathrm{~mm}$ diameter [small diameter $(\mathrm{SmD})]$ or $6.35 \mathrm{~mm}$ diameter [large diameter $(\mathrm{LgD})]$ was inserted between the patellar tendon and tibiofemoral joint or latex band and wood block and the scanning was repeated. Finally, the initial rod was removed, the alternate diameter rod was inserted, and the scanning was repeated. CT imaging was used for point tracking since our $3 \mathrm{D}$ motion tracking hardware was not capable of uniquely identifying the small beads used. Following image acquisition and bead segmentation, the finite strain between the centroid of the imaged beads was calculated between the baseline and small rod and the baseline and large rod configurations $(31,32)$. The mean $( \pm$ SD) maximum principal strain across all samples (cadaveric and physical models) was $7.4 \% \pm 5.4 \%$ for the $\mathrm{SmD}$ rod, and $11.7 \% \pm 7.1 \%$ for the $\mathrm{LgD}$ rod.

\section{Subjects/study design}

The overall experimental design with designation of surgical groups and time points of evaluation is displayed in Figure 1. Twenty-six male skeletally mature Beagle dogs (52 total limbs), of typical weight $[11.6 \pm 1.8$ (range:

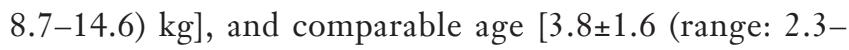
6.8) years] with no history of lameness or experimental drug administration were evaluated. This study was approved by the Cornell University Animal Care and Use Program (protocol \#2014-0123) under the Animal Welfare Assurance A3347-01 in compliance with the Office of Laboratory Animal Welfare (OLAW). Of the original 26 subjects
(52 limbs), 2 subjects (4 limbs) from the control group were removed from the study due to technical challenges of the MRI at the time of examination preventing image data acquisition, and 1 subject ( 2 limbs) from the low strain test group and 1 subject ( 2 limbs) from the high strain test group (a total of 2 subjects and 4 limbs) had MRI acquired, but histologic analysis was not performed. In summary, of the original 26 subjects (52 limbs) a total of 22 subjects (44 limbs) were processed for further evaluation. Using Mead's resource equation method for a pilot animal study, three strain groups and three-time point groups required between 14 and at most 24 canines $(33,34)$ to evaluate differences between strain and time point. Animals were provided housing, food, and enrichment, and monitored according to the Cornell University Animal Care and Use Procedure entitled "Dog Husbandry". Cornell University Hospital for Animal's (CUHA) veterinary anesthesiology staff and Center for Animal Resources and Education (CARE) provided standard anesthesia and perioperative medications based on a combination of drug plans and professional judgment outlined in institutional protocols. The surgical site was prepared by clipping hair and scrubbing with chlorhexidine scrub solution. Vital signs were monitored with the following: capnography, EKG, pulse oximetry, indirect or direct blood pressure, and temperature. A dose of cefazolin $(22 \mathrm{mg} / \mathrm{kg}$ IV) was repeated every 2-3 hours during the surgical procedure. A povidone-iodine scrub and $70 \%$ isopropyl alcohol or $70 \%$ ethanol-soaked gauze sponges were used for surgical 


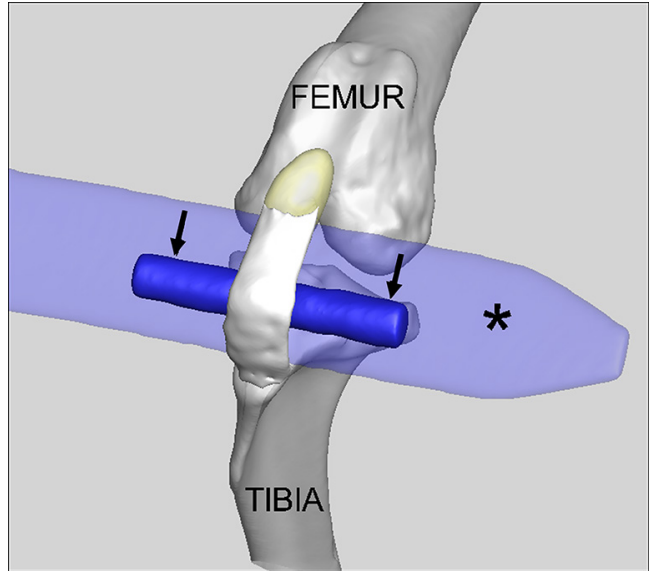

Figure 2 Schematic diagram of the surgical procedure to induce strain in the canine patellar tendon. A stabilizing plate $\left(^{*}\right)$ was placed between the femur and tibia in an open procedure, and stainless-steel rod (arrows) of either 6.35 or $4.76 \mathrm{~mm}$ diameter was placed between the stabilizing plate and patellar tendon to strain the tendon.

Table 1 MRI acquisition parameters

\begin{tabular}{|c|c|c|}
\hline \multirow{2}{*}{ Variables } & \multicolumn{2}{|c|}{ Scanning series } \\
\hline & UTE & PD FSE \\
\hline TE (ms) & $\begin{array}{c}0.03,0.6,1.2,1.8,2.4 \\
3.0,3.6,4.2,4.8,5.4,6.0 \\
6.6,7.2,7.8,8.6,9.0\end{array}$ & 25 \\
\hline $\mathrm{TR}(\mathrm{ms})$ & 99.4 & 4,000 \\
\hline Field-of-view (cm) & 12 & 11 to 14 \\
\hline Flip angle (deg) & 13 & 142 \\
\hline Acquisition matrix & $256 \times 256$ & $416 \times[320-384]$ \\
\hline Slice thickness $(\mathrm{mm})$ & 3 & 2.0 to 2.5 \\
\hline $\mathrm{SS}(\mathrm{mm})$ & 0 & 0 \\
\hline NEX & 1 & 1.5 \\
\hline ETL & 1 & 13 \\
\hline $\begin{array}{l}\text { Acquisition } \\
\text { bandwidth }(\mathrm{kHz})\end{array}$ & \pm 125 & \pm 63 \\
\hline
\end{tabular}

MRI, magnetic resonance imaging; UTE, ultrashort echo; PD, proton density; FSE, fast-spin echo; TE, echo time; TR, repetition time; SS, slice spacing; NEX, number of excitations; ETL, echo train length.

site preparation and painted with a $10 \%$ povidone-iodine or chlorhexidine solution and covered with sterile drapes.
A method previously used by our group for creating simulated strain/sprain at the midsubstance of a ligament $(35,36)$, was adapted for an orthopaedic model of canine patellar tendinopathy. The patellar tendon was elongated in an attempt to create tendon damage at the inferior pole of the patella (35), similar to what is seen clinically in humans $(37,38)$. The resulting effects are comparable to other methods of inducing mechanical fatigue damage $(13,39)$. This method may also localize tendon damage, which is a known challenge to implement with chemical/ enzymatic degradation (40-42). Surgery was performed in the morning, with the animals only permitted cage activity prior to surgery and after surgery. Animals indicated for histologic outcomes only underwent a second procedure for minimally invasive biopsy of the tendon.

Following surgical site preparation in a sterile field, a SmD or LgD stainless-steel rod was passed deep to the midsubstance of the patellar tendon to distract and elongate the patellar tendon to create tendon damage (Figure 2). The strain limb was not randomized, but the choice of a $\mathrm{LgD}$ or $\mathrm{SmD}$ rod and the laterality of the imposed strain were chosen at random until the options were exhausted. The different diameters were used to induce two distinct levels of strain and evaluate the resulting exposure-response on the MRI outcome metrics. The incision site was closed following damage creation, and the animals were permitted free cage movement after recuperation with no additional exercise protocol implemented. Each limb was evaluated at 4 or 8 weeks post-operatively (Figure 1). Four of the 14 test subjects ( 8 limbs) were euthanized and used for biomechanical analysis since in vivo testing protocol load to failure was evaluated. One of the biomechanical testing limbs was removed from the analysis as there was a rupture present, leaving 7 total limbs available for biomechanical analysis. The limbs were selected at random based on availability of MRI data, with a larger number of limbs were selected from the $\mathrm{LgD}$ group in anticipation of larger effects being detected across the MRI, histologic and biomechanical testing outcome measures. All other limbs in the testing category underwent punch biopsy of the patellar tendon for histology at the indicated time point.

\section{MRI}

In vivo scanning was performed (Table 1 ) on a $3 \mathrm{~T}$ clinical imaging system (DVMR 750, GE Healthcare, Milwaukee WI, USA), using an eight channel transmit/receive (T/R) 
knee coil (Invivo, Gainesville, FL, USA) with the subjects in sternal recumbency and the patellar tendons parallel to $\mathrm{B}_{0}$. Multi-planar morphologic FSE images were acquired with the following parameters: echo time (TE): $25 \mathrm{~ms}$, repetition time (TR): 4,500 ms, echo train length (ETL): 12 , receiver bandwidth (RBW): $\pm 83.3 \mathrm{kHz}$, voxel size (VS): $0.26 \times 0.26 \times 1.5 \mathrm{~mm}^{3}$, slice spacing (SS): $0 \mathrm{~mm}$, number of excitations (NEX): 3. A sagittal 3D CONES UTE series (43) was acquired with the parameters: 16 echoes: TEs $=0.03$ to $9.0 \mathrm{~ms}$, with $\Delta \mathrm{TE}=0.6 \mathrm{~ms}$, flip angle: $15^{\circ}$, TR: $25 \mathrm{~ms}$, VS: $0.38 \times 0.38 \times 3 \mathrm{~mm}^{3}, \mathrm{RBW}: \pm 125 \mathrm{kHz}, \mathrm{NEX}: 1$. In addition, frequency selective fat saturation was utilized with five read outs per single excitation. Short $\mathrm{T}_{2} *(\mathrm{~T} 2 * \mathrm{~S})$ and long $\mathrm{T} 2$ * $(\mathrm{T} 2 * \mathrm{~L})$ values of the tendon signal decay were calculated to evaluate the bound (short) and free (long) water components of the tendon (44), respectively, on a voxel-by-voxel basis. SI was modeled across the 16 TEs as a biexponential decay function using custom written software (MATLAB, Natick, MA, USA):

$$
S I(T E)=A \cdot e^{-\frac{T E}{T 2^{*} S}}+B \cdot e^{-\frac{T E}{T 2^{*} L}}+C
$$

Where the fitting coefficients $\mathrm{A}$ and $\mathrm{B}$ are used to calculate the percent contribution of the short $[\mathrm{PS}=\mathrm{A} / \mathrm{A}$ $+\mathrm{B})]$ and long $[\mathrm{PL}=\mathrm{B} /(\mathrm{A}+\mathrm{B})]$ components of the overall signal decay, and $\mathrm{C}$ is used to account for the image noise floor (44). A global (full tendon) T2* variable analysis was performed.

\section{Histology}

For a subgroup of specimens (Figure 1) the patellar tendon was extracted and fixed in $4 \%$ neutral buffered paraformaldehyde, paraffin embedded, and $6 \mu \mathrm{m}$ sections stained using Safranin-O fast green for matrix metachromasia and extent of vascular penetration measures using Scion Image Beta 4.03. Picrosirius red staining with polarized light microscopy evaluation was used to assess collagen orientation and maturation (45), and H\&E of longitudinal sections of the patellar tendon were evaluated (46). Four separate tendon features were graded from 0 (best) to 3 (worst): (I) tenocyte (spindle cell) morphology and proliferation, (II) presence or absence of ground substance, (III) collagen bundle characteristics, and (IV) vascularity.

\section{Biomechanical testing}

For a subgroup of specimens (Figure 1), the limbs were wrapped and immediately frozen at $-80{ }^{\circ} \mathrm{C}$ until testing performed using a previously described method (47). Briefly, the specimens were thawed at room temperature for 12 hours prior to preparation, after which the musculature and other soft tissues were carefully dissected from the stifles to isolate the quadriceps tendon, patella, patellar tendon and tibia as a single unit. The tibial shaft was secured in bone cement, and a custom clamp was secured around the tendon just below the distal pole of the patella. The tibia was position at 20 degrees from the vertical. The specimen was initially loaded to $10 \mathrm{~N}$ to remove residual slack from the patellar tendon and the width and thickness at the midsubstance of the patellar tendon were measured three times by a single author (MFK) to calculate the cross-sectional area of the tendon assuming a rectangular cross section. The initial length of the tendon from the tibial tuberosity to the clamp was also measured three times and averaged. Finally, retro-reflective markers were attached to the tendon clamp, tendon mid-substance, tibia, and tibial clamp by using a small amount of cyanoacrylate to utilize a non-contact optical measuring system (Motion Analysis Corporation, Rohnert Park, CA, USA) to measure displacements. The loading protocol consisted of ramp loading the specimen to $50 \mathrm{~N}$ over 10 seconds, followed by preconditioning the specimen using cyclic loading between 50 and $180 \mathrm{~N}$ for 20 cycles at $1 \mathrm{~Hz}$, and completed by loading the specimen to failure at $100 \%$ strain/s. The outcome variables from the testing were modulus, failure stress, and failure strain and were determined using the strain measured between the tendon mid-substance and the tibia.

\section{Statistical analysis}

MRI, histology, and biomechanical outcomes were summarized with medians and interquartile ranges (IQRs). Median differences between control, SmD and $\mathrm{LgD}$ strain group and between 0,4 , and 8 -week limbs were estimated using quantile regression with cluster robust $95 \%$ percentile bootstrap confidence intervals (CIs) calculated from 1,000 resamples to account for limbs from the same beagle. Since we cannot generate data under a null hypothesis with the bootstrap method which is necessary to calculate a $\mathrm{P}$ value, statistical significance of the difference is implied by bootstrapped $95 \%$ CIs that exclude the null value of 0 . In addition, a minimum clinically important difference (MCID) (48) of $0.24 \mathrm{~ms}$ was defined to interpret the resulting differences of $\mathrm{T} 2 * \mathrm{~S}$ based on previous reports in the literature $(44,49,50)$. Analyses were performed in SAS version 9.4 (SAS Institute Inc., Cary, NC, USA). 
Table 2 MRI outcomes by level of induced strain

\begin{tabular}{|c|c|c|c|c|c|c|}
\hline Variables & \multicolumn{3}{|c|}{ Induced strain level $^{\star}$} & \multicolumn{3}{|c|}{ Differences $^{\star \star}$} \\
\hline $\mathrm{N}$ & 10 & 5 & 8 & & & \\
\hline $\mathrm{T} 2 * \mathrm{~S}(\mathrm{~ms})$ & $0.5[0.3,0.6]$ & $0.9[0.6,1.0]$ & $0.8[0.7,0.9]$ & $-0.2(-0.3,0.3)$ & $0.3(0.1,0.5)$ & $0.5(0.0,0.7)$ \\
\hline $\begin{array}{l}\text { Percent contribution } \\
\text { long (\%) }\end{array}$ & $60.1[53.0,69.0]$ & $60.6[58.6,61.0]$ & $63.6[59.5,66.0]$ & $3.0(-1.3,5.5)$ & $3.5(-7.6,12.9)$ & $0.5(-10.4,9.2)$ \\
\hline
\end{tabular}

*, Values displayed as median [IQR]; **, values displayed as difference with (95\% bootstrapped Cl). MRI, magnetic resonance imaging; $\mathrm{IQR}$, interquartile range; $\mathrm{Cl}$, confidence interval.

Table 3 MRI outcomes by post-operative time point

\begin{tabular}{|c|c|c|c|c|c|c|}
\hline Variables & \multicolumn{3}{|c|}{ Time point* } & \multicolumn{3}{|c|}{ Differences ${ }^{* *}$} \\
\hline $\mathrm{N}$ & 10 & 4 & 9 & & & \\
\hline $\mathrm{T} 2 * \mathrm{~S}(\mathrm{~ms})$ & $0.5[0.3,0.6]$ & $1.0[0.9,1.2]$ & $0.7[0.6,0.8]$ & $-0.3(-0.5,-0.1)$ & $0.3(0.1,0.5)$ & $0.5(0.3,0.8)$ \\
\hline $\mathrm{T} 2 * \mathrm{~L}(\mathrm{~ms})$ & $4.2[3.7,4.8]$ & $6.4[6.2,7.7]$ & $4.6[3.7,4.8]$ & $-1.8(-3.9,-0.8)$ & $0.4(-1.0,1.6)$ & $2.2(1.2,3.9)$ \\
\hline $\begin{array}{l}\text { Percent contribution long } \\
\text { (\%) }\end{array}$ & $60.1[53.0,69.0]$ & $65.6[58.8,66.2]$ & $60.8[58.6,62.0]$ & $-4.8(-7.5,2.9)$ & $0.7(-9.4,8.4)$ & $5.5(-9.9,12.9)$ \\
\hline
\end{tabular}

*, values displayed as median [IQR]; **, values displayed as difference with (95\% bootstrapped Cl). MRI, magnetic resonance imaging; $\mathrm{IQR}$, interquartile range; $\mathrm{Cl}$, confidence interval.

\section{Results}

\section{$q M R I$}

The SmD and $L g D$ strain groups had prolongation of median (IQR) of $\mathrm{T}^{*}{ }^{*} \mathrm{~S}$ as compared to the control group [control: $0.5(0.3,0.6) \mathrm{ms}$; SmD strain: $0.9(0.6,1.0) \mathrm{ms}$; LgD strain: $0.8(0.7,0.9) \mathrm{ms}]$. The median difference of $\mathrm{T}^{*} \mathrm{~S}$ value between the $\mathrm{LgD}$ and control groups was greater than the MCID (Table 2), and the 95\% CI excluded zero. No differences of PS and PL were detected between the control and $\mathrm{SmD}$ or $\mathrm{LgD}$ strain groups. Larger differences of MRI metrics were detected between control limbs and the limbs at 4 and 8 weeks. At 4 weeks, the difference of $\mathrm{T} 2 * \mathrm{~S}, 0.5(0.3,0.8) \mathrm{ms}$, was greater than the MCID as compared to controls, and the $95 \%$ CI excluded zero. At 8 weeks, $\mathrm{T} 2{ }^{*}$ s was reduced as compared to 4 weeks [median difference: $-0.3(-0.5,-0.1) \mathrm{ms}$, with prolongation as compared to the control limbs [median difference: 0.3 $(0.1,0.5) \mathrm{ms}$, Table 3 and Figure 3].

\section{Histology}

Histology of the $\mathrm{LgD}$ strain group displayed greater collagen disruption $[\mathrm{SmD}: 2(1,3), \operatorname{LgD}: 3(0,3)]$, lower cellularity $[\mathrm{SmD}: 2(1,3), \operatorname{LgD}: 1(0,2)]$, and decreased vascularity $[\mathrm{SmD}: 2(0,2), \operatorname{LgD}: 1(0,2)]$ as compared to the SmD strain group (Table 4). The $\mathrm{LgD}$ strain group also had greater mean birefringence [SmD: 19.3 (18.0, 25.6), LgD: $22.0(17.4,53.9)]$ and greater percent birefringence [SmD: $5.4(3.9,8.2), \operatorname{LgD}: 8.6(1.9,16.8)]$ than the $\mathrm{SmD}$ strain group. A representative histologic comparison of tendons at 4 and 8 weeks post-operatively is shown in Figure 4. 

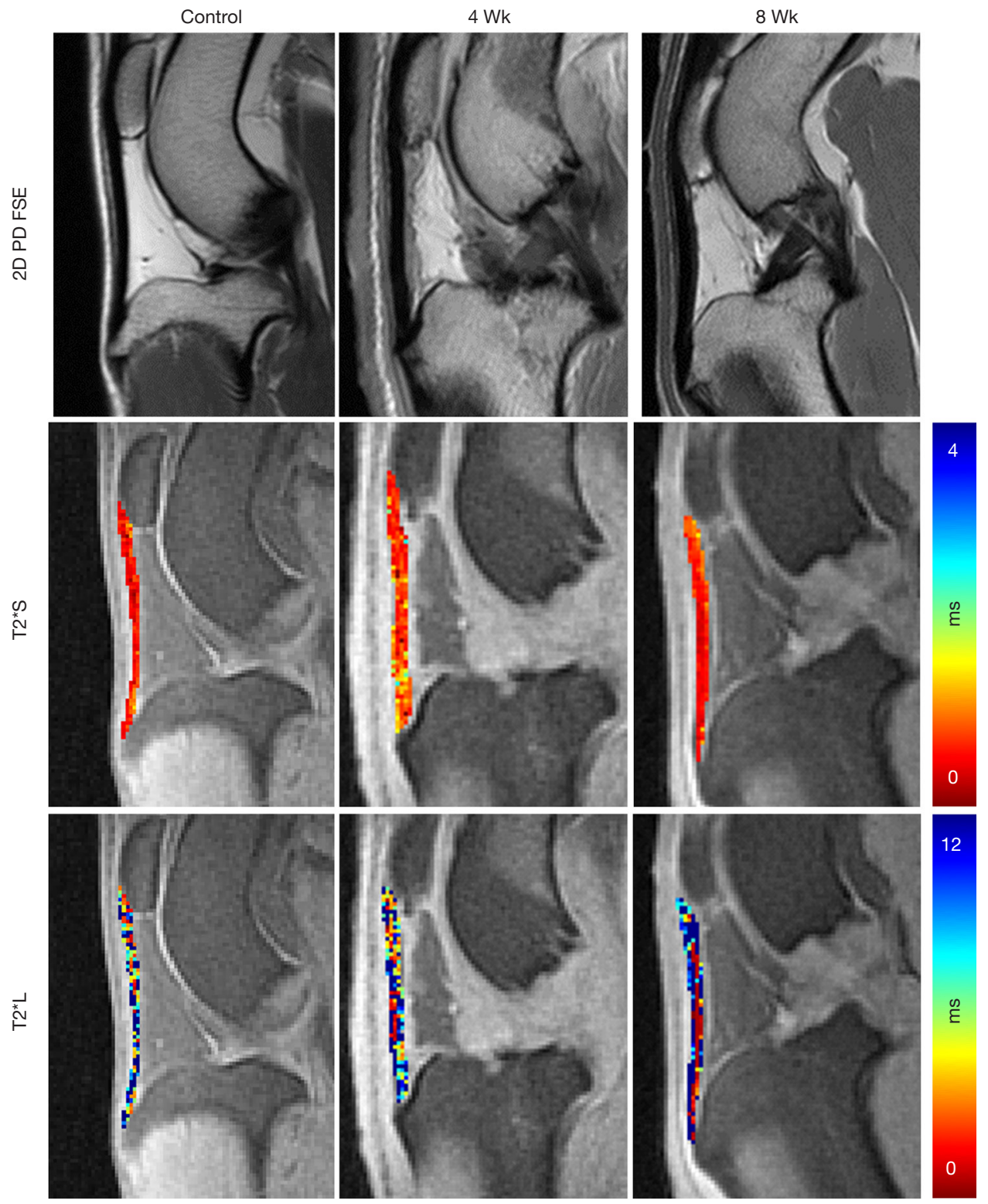

Figure 3 Morphologic PD fast-spin-echo images (top row), short $\mathrm{T}^{*}$ component map (T2*S, middle row), and long $\mathrm{T} 2{ }^{*}$ component map (T2* ${ }^{*}$, bottom row) of a control patellar tendon and a patellar tendon at 4 and 8 weeks post-operatively after induction of mechanical strain. The PD images displayed no differences in SI between the control limb and each time point, while prolongation of T2* was seen at 4 weeks which subsided at 8 weeks. Great variability of T2*L was seen within each tendon. PD, proton density; SI, signal intensity; FSE, fast-spin echo.

Histology of the 8 -week group displayed lower cellularity [4-week: $1.5(1,2.5), 8$-week: $1(0,2.5)$ ], reduced collagen disruption [4-week: $3(1.5,3), 8$-week: $1(0,2.5)]$, decreased granulation tissue [4-week $1.5(0.5,2), 8$-week $0(0,3)$ ], and reduced tenocyte populations [4-week: $1(1,2), 8$-week: $0.5(0,1)]$, as compared to the 4 -week group. The 8 -week samples also had larger values for mean birefringence [4-week:
20.7 (18.2, 24.3), 8-week: $22.0(12.2,70.9)]$ and percent birefringence [4-week: $4.5(2.9,10.9), 8$-week: $7.3(4.6,15.8)]$.

\section{Biomechanics}

The SmD and $\mathrm{LgD}$ strain levels affected the mechanical properties of the patellar tendon. Both levels resulted in a 
Table 4 Histologic outcomes by level of induced strain and time point

\begin{tabular}{|c|c|c|c|c|c|c|}
\hline Variable evaluated & \multicolumn{3}{|c|}{ Induced strain level ${ }^{\star}$} & \multicolumn{3}{|c|}{ Time point ${ }^{\star}$} \\
\hline Cellularity & $2[1,3]$ & $1[0,2]$ & $-1[-2,1]$ & $1.5[1,2.5]$ & $1[0,2.5]$ & $-0.5[-2.5,2]$ \\
\hline Collagen & $2[1,3]$ & $3[0,3]$ & $1[-2,2.5]$ & $3[1.5,3]$ & $1[0,2.5]$ & $-2[-3,0]$ \\
\hline Granulation & $1[0,2]$ & $1[0,2]$ & $0[-2,2]$ & $1.5[0.5,2]$ & $0[0,3]$ & $-1.5[-2,2]$ \\
\hline Tenocytes & $1[0,1]$ & $1[0,1]$ & $0[-1,1]$ & $1[1,2]$ & $0.5[0,1]$ & $-0.5[-2,0]$ \\
\hline Vascularity & $2[0,2]$ & $1[0,2]$ & $-1[-2,1]$ & $1[1,2.5]$ & $1[0,2]$ & $0[-3,1]$ \\
\hline Mean birefringence & $19.3[18,25.6]$ & $22[17.4,53.9]$ & $2.7[-49.9,35.9]$ & $20.7[18.2,24.3]$ & $22.0[12.2,70.9]$ & $1.6[-9.3,51.7]$ \\
\hline Percent birefringence (\%) & $5.4[3.9,8.2]$ & $8.6[1.9,16.8]$ & $3.2[-4.1,11.4]$ & $4.5[2.9,10.3]$ & $7.3[4.6,15.8]$ & $2.8[-2.8,13.1]$ \\
\hline
\end{tabular}

${ }^{*}$, values displayed as median [IQR]; ${ }^{*}$, values displayed as difference with [95\% bootstrapped $\left.\mathrm{Cl}\right]$. IQR, interquartile range; Cl, confidence interval.
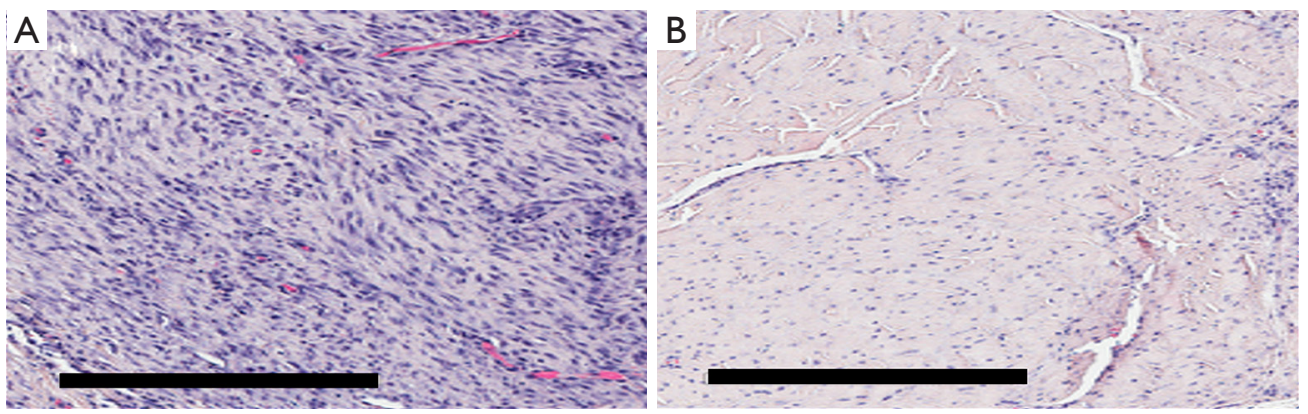

Figure 4 Four- (A) and 8-week (B) H\&E histology of the patellar tendon with LgD strain. Notice at 4 weeks, the presence of immature fibroblasts, hyper-cellular infiltration, limited collagen production and evidence of localized remodeling. At 8 weeks, there is reduced cellularity, populations of fibroblasts and mature fibrocytes, and increased collagen production. Bar $=300$ microns. $\mathrm{LgD}$, large diameter.

lower failure stress as compared to control limbs [median difference of SmD: $-11.7(-24.9,-5.7) \mathrm{MPa}$; median difference of $\operatorname{LgD}:-11.3(-26.0,-4.2) \mathrm{MPa}$, Table 5]. The $\mathrm{LgD}$ strain group had a lower modulus than the control group [median difference: $-807.3(-990.2,-0.1) \mathrm{MPa}$ ]. Differences in mechanical properties were also detected over time. The 4-week group had a greater failure strain than the control group [median difference: $1.2 \%(1.0 \%$, $4.5 \%)$ ] and the 8 -week group [median difference: $1.9 \%$ $(1.9 \%, 4.0 \%)]$. The failure stress at 4 and 8 weeks was lower than control limbs [median difference at 4 weeks: -13.1 $(-27.0,-1.9) \mathrm{MPa}$; median difference at 8 weeks: $-8.4(-26.0$, $-5.3) \mathrm{MPa}$. The failure stress at 4 and 8 weeks were similar. The modulus at 4 weeks was less than control limbs [median difference: $-841.1(-1,020.6,-294.2) \mathrm{MPa}$. The crosssectional area at 4 weeks was greater than the control limbs [median difference: $26.0(5.2,44.6) \mathrm{mm}^{2}$ ].

\section{Discussion}

Noninvasive imaging methods exist to evaluate the spatial distribution of patellar tendon injury as well as temporal changes within the tendon during the healing/ remodeling process. However, standard evaluation depends on interpretation of morphologic images. Newer qMRI techniques, such as $\mathrm{T} 2{ }^{*}$ mapping using UTE presented herein, permit an objective, quantitative approach to the in vivo assessment of tendon health and healing. This pilot study evaluated the effects of an induced mechanical strain of the patellar tendon in a canine model on indirect quantitative methods of UTE-MRI metrics as well as direct methods of biomechanical and histologic evaluation. 
Table 5 Biomechanical outcomes of induced strain and time point

\begin{tabular}{|c|c|c|c|c|c|c|}
\hline Variables & \multicolumn{3}{|c|}{ Induced strain level } & \multicolumn{3}{|c|}{ Time point } \\
\hline $\begin{array}{l}\text { Cross-sectional area } \\
\left(\mathrm{mm}^{2}\right)\end{array}$ & $\begin{array}{c}12.9 \\
{[12.0,15.5]}\end{array}$ & $\begin{array}{c}36.3 \\
{[18.4,54.2]}\end{array}$ & $\begin{array}{c}26.0 \\
{[25.6,51.8]}\end{array}$ & $\begin{array}{c}12.9 \\
{[12.0,15.5]}\end{array}$ & $\begin{array}{c}38.9 \\
{[25.8,53.0]}\end{array}$ & $\begin{array}{c}18.4 \\
{[16.2,53.2]}\end{array}$ \\
\hline $\begin{array}{l}\text { Energy to failure } \\
(\mathrm{N}-\mathrm{mm})\end{array}$ & $\begin{array}{c}409.6 \\
{[184.0,507.2]}\end{array}$ & $\begin{array}{c}159.0 \\
{[110.8,207.3]}\end{array}$ & $\begin{array}{c}226.4 \\
{[114.3,268.2]}\end{array}$ & $\begin{array}{c}409.6 \\
{[184.0,507.2]}\end{array}$ & $\begin{array}{c}189.5 \\
{[97.3,760.7]}\end{array}$ & $\begin{array}{c}207.3 \\
{[114.3,226.4]}\end{array}$ \\
\hline Failure strain (\%) & $\begin{array}{c}4.4 \\
{[2.5,4.9]}\end{array}$ & $\begin{array}{c}3.5 \\
{[2.3,4.8]}\end{array}$ & $\begin{array}{c}4.8 \\
{[4.6,6.3]}\end{array}$ & $\begin{array}{c}4.4 \\
{[2.5,4.9]}\end{array}$ & $\begin{array}{c}5.6 \\
{[4.8,8.2]}\end{array}$ & $\begin{array}{c}3.7 \\
{[2.3,4.6]}\end{array}$ \\
\hline Failure stress (MPa) & $\begin{array}{c}27.0 \\
{[22.4,32.9]}\end{array}$ & $\begin{array}{c}15.3 \\
{[12.0,18.7]}\end{array}$ & $\begin{array}{c}15.7 \\
{[10.9,20.8]}\end{array}$ & $\begin{array}{c}27.0 \\
{[22.4,32.9]}\end{array}$ & $\begin{array}{c}13.9 \\
{[9.9,22.4]}\end{array}$ & $\begin{array}{c}18.7 \\
{[10.9,20.8]}\end{array}$ \\
\hline
\end{tabular}

All values displayed as median [IQR]. IQR, interquartile range.

The results found that the induced strain damage to the patellar tendon affected MRI metrics. A prolongation of $\mathrm{T} 2{ }^{*} \mathrm{~S}, 108 \%$ greater than our defined MCID, was found between the SmD strain group and the control limbs, while a smaller prolongation of $\mathrm{T} 2 * \mathrm{~S}(+25 \%)$ was found between the $\mathrm{LgD}$ strain group and control limbs. Similarly, a large $(+100 \%)$ difference of $\mathrm{T} 2{ }^{*} \mathrm{~S}$ was found between the control limbs and 4 -week time points. $\mathrm{T} 2{ }^{*} \mathrm{~S}$ was still prolonged at 8 weeks post-operatively with a smaller $(60 \%)$ increase in magnitude as compared to control limbs. The difference of $\mathrm{T} 2{ }^{*} \mathrm{~S}$ was greater than the MCID at both post-operative time points. The percent difference between the $\mathrm{LgD}$ and $\mathrm{SmD}$ groups is unclear but may be attributable the limited sample size or the subject specific response to the imposed strain since the results of our different strain validation experiments only found a $4.3 \%$ strain difference between the $\mathrm{SmD}$ and $\mathrm{LgD}$ groups. In addition, a biexponential fitting method, rather than a mono-exponential method, was used in the current study due to previous reports of the higher sensitivity of the short component when pathologic differences are present (44).

We believe that the differences that display prolongation of $\mathrm{T} 2{ }^{*} \mathrm{~S}$ at 4 weeks and a shortening by 8 weeks provides insight into the healing time course of the damaged tendon by a qMRI technique. While $\mathrm{T} 2{ }^{*} \mathrm{~S}$ at 8 weeks was still prolonged as compared to control specimens, it was reduced in magnitude when compared to limbs at 4 weeks, indicative of greater collagen fibril organization, as corroborated by the corresponding histologic evaluation. We also note that the percent difference of $\mathrm{T} 2{ }^{*} \mathrm{~S}$ detected between the control limbs and limbs at 4 weeks post-op are similar in magnitude to those previously described in the literature in the setting of tendinopathy due to overuse or mechanical injury $(44,50)$.

Our analysis also found large variability of $\mathrm{T} 2 * \mathrm{~L}$ for each strain level and at each time point. This result is similar to what has been reported in the literature for the human Achilles and patellar tendons $(44,51)$ The variability of $\mathrm{T} 2{ }^{*} \mathrm{~L}$ may be attributable to the local water content concomitant with collagen fibril orientation and density. Regions with highly ordered collagen fibrils will have an initial rapid signal decay due to short $\mathrm{T}^{*} \mathrm{~S}$, which may in turn accentuate the influence of noise on the calculated $\mathrm{T} 2{ }^{*} \mathrm{~L}$ value. Further evaluation of the long component using advanced data evaluation techniques to minimize the influence of noise (52) would be beneficial to incorporate in future studies, as well as determining if the patellar tendon may be best examined using a mono-exponential fit as has been done for the Achilles tendon (44).

The corresponding histologic analysis and biomechanical testing had similar findings based on induced strain level and time point of evaluation. Strained specimens had increased cellularity and disorganization of collagen fibrils, in addition to greater cross-sectional area and reduced mechanical properties, indicative of patellar tendinopathy. Greater collagen disruption in the $\mathrm{LgD}$ group was seen in the histologic samples, but a corresponding prolongation of $\mathrm{T} 2{ }^{*} \mathrm{~S}$ was not evident. We attribute this lack of correspondence to the small sample size of the study and the greater histologic variation that was displayed in the $\mathrm{LgD}$ group. Notably, the histologic analysis found improvement 
of tendons indicated by reduced levels of cellularity and collagen disorganization at 8 weeks as compared to 4 weeks. The biomechanical analysis found greater differences in modulus, failure stress and failure strain between the control limbs and limbs at 4 weeks as compared to control limbs and limbs at 8 weeks. These results indicate that the tendons are undergoing healing in response to the imposed strain. Similarly, the rate difference of $T 2 * S$ likely indicates the rate of tendon healing, although this is challenging to confirm in the current pilot study with only two-time points and a limited number of limbs. Evaluating the longterm longitudinal maturation of $\mathrm{T} 2{ }^{*} \mathrm{~S}$ with corresponding histology would be necessary for verification.

The constellation of findings at the early and later time points reflects a gradual trend toward more organized tendon and tissue healing, indicative of reduced longitudinal tendinopathy. The results indicate the capability of utilizing quantitative T2* UTE MRI to evaluate differences in tissue response to load, associating imaging parameters to mechanical damage. The qMRI technique of UTE T2* mapping may be clinically useful in the setting of a normal and strained tendon to evaluate the effectiveness of different rehabilitation protocols aimed at promoting tendon healing and further to eventually develop predictive models of recovery, and in the athletic population, return to play.

This study had several limitations. First, the midsubstance of the patellar tendon was the primary location of the induced mechanical damage rather than a superior and posterior location as seen in most cases of patellar tendinopathy, or an inferior location commonly seen in adult Osgood-Schlatter disease $(53,54)$. Inducing damage at the mid-substance was facilitated by being able to restrain motion of the patella and tibial to ensure that the strain was applied. Our prior attempts to apply the strain closer to the distal pole of the patella resulted in noticeable patellar motion. It would be beneficial to have a device that could aid in positioning and loading of the patellar tendon for greater uniformity of applied load to induce damage. Second, as this was a pilot study, the sample size was a limiting factor in addition to the single time point MRI evaluation per subject due to the necessity of tissue sampling for histology and/or biomechanical testing. Future studies would benefit from a larger number of samples for analysis for each procedure and at each time point. In addition, in vivo scanning of the limbs would permit longitudinal evaluation of changes in the tendon rather than differences of separate specimens between time points. Third, it would be beneficial to assess the reproducibility of the MRI outcomes used in the current study, as understanding measurement repeatability facilitates interpretation of study results, but we note that previous investigators have found low coefficients of variation $(\mathrm{CoV}: \sim 6-11 \%)$, excellent repeatability [intraclass correlation coefficient (ICC): $\sim 0.98$ ], and minimal test-retest differences of $\mathrm{T}^{*}$ metrics $(\sim 3.8 \%$ for $\left.\mathrm{T} 2{ }^{*} \mathrm{~S}\right)$ using comparable UTE imaging techniques and analysis methods used in the current study $(44,49,50,55)$. Finally, only strained tendons had histologic assessment performed, and were only graded for morphology. Assessing the extent and magnitude of patellar tendinopathy resulting from the imposed strain as well as the time course of healing as compared to unaffected control limbs would be beneficial. In addition, more advanced quantitative histologic techniques, such as polarized light microscopy, may provide a means for a direct comparison of local collagen organization and disruption with corresponding local $\mathrm{T}^{*}$ metrics.

In summary, in this study we identified differences in $\mathrm{T}^{*}$ during healing of induced patellar damage in a canine model, suggesting further investigation into $\mathrm{T} 2{ }^{*}$ maps from UTE images as a quantitative metric for evaluation of tendon healing. Standard morphological MRI typically displays the patellar tendon but is unable to provide insight into tendon structure. T2* mapping from UTE MRI has been used as a biomarker for organization and/ or degeneration in highly ordered collagenous structures such as tendon, but a longitudinal evaluation of the patellar tendon, in humans or an animal model, has not been performed to date. The results of this pilot study indicate that differences in the MRI parameter $\mathrm{T}^{*}$ may be identified during the course of tendon healing, and these imaging metrics are correlated with histological and biomechanical testing. In addition, a canine model of patellar tendinopathy is beneficial as the large anatomy affords the ability to utilize standard clinical imaging equipment, as was done in this study. Future studies will benefit from canine training to run on a treadmill, sit and stand with weights, and walk over a force plate for objective gait analysis of the same animal over time (19).

\section{Acknowledgments}

Funding: Hospital for Special Surgery MRI Laboratory receives institutional research support from GE Healthcare. Internal institutional financial support from the Coleman MRI Research Fund was used to perform this study. 


\section{Footnote}

Conflicts of Interest: All authors have completed the ICMJE uniform disclosure form (available at http://dx.doi. org/10.21037/qims-20-684). Dr. SLP and Dr. REB report other from GE Healthcare, outside the submitted work. Dr. MFK reports other from GE Healthcare, other from GE/NBA, grants from NIH, grants from NIH NIAMS, outside the submitted work. Dr. HGP reports other from GE Healthcare, other from GE/NBA, grants from National Institutes of Health (NIAMS/NICHD), personal fees from Ortho RTI, personal fees from Stryker, outside the submitted work, and Member of Editorial or Governing boards of: AOSSM, Imaging in Sports Health, Fournal of Hip Preservation Surgery, Fournal of Orthopaedic Research, Osteoarthritis and Cartilage; owns stock or stock options in: Imagen. The other authors have no conflicts of interest to declare.

Ethical Statement: This study was approved by the Cornell University Animal Care and Use Program (protocol \#20140123) in compliance with the Animal Welfare Assurance A3347-01 on file with the Office of Laboratory Animal Welfare (OLAW).

Open Access Statement: This is an Open Access article distributed in accordance with the Creative Commons Attribution-NonCommercial-NoDerivs 4.0 International License (CC BY-NC-ND 4.0), which permits the noncommercial replication and distribution of the article with the strict proviso that no changes or edits are made and the original work is properly cited (including links to both the formal publication through the relevant DOI and the license). See: https://creativecommons.org/licenses/by-nc-nd/4.0/.

\section{References}

1. Khan KM, Maffulli N, Coleman BD, Cook JL, Taunton JE. Patellar tendinopathy: some aspects of basic science and clinical management. Br J Sports Med 1998;32:346-55.

2. Khan KM, Maffulli N. Tendinopathy: an Achilles' heel for athletes and clinicians. Clin J Sport Med 1998;8:151-4.

3. Karlsson J, Lundin O, Wigerstad I, Peterson L. Partial rupture of the Results after patellar ligament. Am J Sports Med 1991;19:403-8.

4. Karlsson J, Kälebo P, Goksör LA, Thomee R, Swärd L. Partial rupture of the patellar ligament. Am J Sports Med 1992;20:390-5.
5. Lian ØВ, Engebretsen L, Bahr R. Prevalence of jumper's knee among elite athletes from different sports: A crosssectional study. Am J Sports Med 2005;33:561-7.

6. Ferretti A, Papandrea P, Conteduca F, Mariani PP. Knee ligament injuries in volleyball players. Am J Sports Med 1992;20:203-7.

7. Cook JL. A cross sectional study of 100 athletes with juniper's knee managed conservatively and surgically. Br J Sports Med 1997;31:332-6.

8. Brockmeyer M, Diehl N, Schmitt C, Kohn DM, Lorbach O. Results of surgical treatment of chronic patellar tendinosis (Jumper's knee): a systematic review of the literature. Arthroscopy 2015;31:2424-9.e3.

9. Mienaltowski MJ, Dunkman AA, Buckley MR, Beason DP, Adams SM, Birk DE, Soslowsky LJ. Injury response of geriatric mouse patellar tendons. J Orthop Res 2016;34:1256-63.

10. Gilday SD, Chris Casstevens E, Kenter K, Shearn JT, Butler DL. Murine patellar tendon biomechanical properties and regional strain patterns during natural tendon-to-bone healing after acute injury. J Biomech 2014;47:2035-42.

11. Fu SC, Yeung MY, Rolf CG, Yung PSH, Chan KM, Hung LK. Hydrogen peroxide induced tendinopathic changes in a rat model of patellar tendon injury. J Orthop Res 2018;36:3268-74.

12. Andarawis-Puri N, Sereysky JB, Jepsen KJ, Flatow EL. The relationships between cyclic fatigue loading, changes in initial mechanical properties, and the in vivo temporal mechanical response of the rat patellar tendon. J Biomech 2012;45:59-65.

13. Fung DT, Wang VM, Andarawis-Puri N, Basta-Pljakic J, Li Y, Laudier DM, Sun HB, Jepsen KJ, Schaffler MB, Flatow EL. Early response to tendon fatigue damage accumulation in a novel in vivo model. J Biomech 2010;43:274-9.

14. Bertollo N, Pelletier MH, Walsh WR. Relationship between patellar tendon shortening and in vitro kinematics in the ovine stifle joint. Proc Inst Mech Eng H 2013;227:438-47.

15. Spindler KP, Clark SW, Nanney LB, Davidson JM. Expression of collagen and matrix metalloproteinases in ruptured human anterior cruciate ligament: an in situ hybridization study. J Orthop Res 1996;14:857-61.

16. Shino K, Horibe S. Experimental ligament reconstruction by allogeneic tendon graft in a canine model. Acta Orthop Belg 1991;57:44-53.

17. Das S, Thorne R, Lorenz ND, Clarke SP, Madden M, 
Langley-Hobbs SJ, Perry KL, Burton NJ, Moores AL, Mosley JR. Patellar ligament rupture in the dog: repair methods and patient outcomes in 43 cases. Vet Rec 2014;175:370.

18. Ricciardi M, Lenoci D. Comparative diagnostic imaging of a partial patellar ligament tear in a dog. Open Vet J 2018;8:160-7.

19. Gustås P, Pettersson K, Honkavaara S, Lagerstedt AS, Byström A. Kinematic and spatiotemporal assessment of habituation to treadmill walking in Labrador retrievers. Acta Vet Scand 2016;58:87.

20. Gold GE, Pauly JM, Macovski A, Herfkens RJ. MR Spectroscopic imaging of collagen: tendons and knee menisci. Magn Reson Med 1995;34:647-54.

21. Robson MD, Gatehouse PD, Bydder M, Bydder GM. Magnetic resonance: an introduction to ultrashort. J Comput Assist Tomogr 2003;27:825-46.

22. Fleming BC, Vajapeyam S, Connolly SA, Magarian EM, Murray MM. The use of magnetic resonance imaging to predict ACL graft structural properties. J Biomech 2011;44:2843-6.

23. Gatehouse PD, Bydder GM. Magnetic resonance imaging of short T2 components in tissue. Clin Radiol 2003;58:1-19.

24. Reichert IL, Benjamin M, Gatehouse PD, Chappell KE, Holmes J, He T, Bydder GM. Magnetic resonance imaging of periosteum with ultrashort TE pulse sequences. J Magn Reson Imaging 2004;19:99-107.

25. Koff MF, Shah P, Pownder S, Romero B, Williams R, Gilbert S, Maher S, Fortier LA, Rodeo SA, Potter HG. Correlation of meniscal T2* with multiphoton microscopy, and change of articular cartilage T2 in an ovine model of meniscal repair. Osteoarthritis Cartilage 2013;21:1083-91.

26. Chu CR, Williams AA, West RV, Qian Y, Fu FH, Do $\mathrm{BH}$, Bruno S. Quantitative magnetic resonance imaging UTE-T2* mapping of cartilage and meniscus healing after anatomic anterior cruciate ligament reconstruction. Am J Sports Med 2014;42:1847-56.

27. Williams A, Qian Y, Golla S, Chu CR. UTE-T2* mapping detects sub-clinical meniscus injury after anterior cruciate ligament tear. Osteoarthritis Cartilage 2012;20:486-94.

28. Nissi MJ, Rieppo J, Töyräs J, Laasanen MS, Kiviranta I, Nieminen MT, Jurvelin JS. Estimation of mechanical properties of articular cartilage with MRI - dGEMRIC, $\mathrm{T} 2$ and $\mathrm{T} 1$ imaging in different species with variable stages of maturation. Osteoarthritis Cartilage 2007;15:1141-8.

29. Kurkijärvi JE, Nissi MJ, Kiviranta I, Jurvelin JS, Nieminen MT. Delayed gadolinium-enhanced MRI of cartilage (dGEMRIC) and T2 characteristics of human knee articular cartilage: topographical variation and relationships to mechanical properties. Magn Reson Med 2004;52:41-6.

30. Lammentausta E, Kiviranta P, Nissi MJ, Laasanen MS, Kiviranta I, Nieminen MT, Jurvelin JS. T2 relaxation time and delayed gadolinium-enhanced MRI of cartilage (dGEMRIC) of human patellar cartilage at 1.5 $\mathrm{T}$ and 9.4 $\mathrm{T}$ : Relationships with tissue mechanical properties. J Orthop Res 2006;24:366-74.

31. Omens JH, Fung YC. Residual strain in rat left ventricle. Circ Res 1990;66:37-45.

32. Villarreal FJ, Waldman LK, Lew YW. Technique for measuring regional two-dimensional finite strains in canine left ventricle. Circ Res 1988;62:711-21.

33. Mead R, Gilmour S, Mead A. Statistical principles for the design of experiments: applications to real experiments. Cambridge: Cambridge University Press, 2012.

34. Festing MF, Altman DG. Guidelines for the design and statistical analysis of experiments using laboratory animals. ILAR J 2002;43:244-58.

35. Provenzano PP, Hayashi K, Kunz DN, Markel MD, Vanderby R Jr. Healing of subfailure ligament injury: comparison between immature and mature ligaments in a rat model. J Orthop Res 2002;20:975-83.

36. Provenzano PP, Heisey D, Hayashi K, Lakes R, Vanderby $\mathrm{R}$ Jr. Subfailure damage in ligament: a structural and cellular evaluation. J Appl Physiol (1985) 2002;92:362-71.

37. Peers KH, Lysens RJ. Patellar tendinopathy in athletes: current diagnostic and therapeutic recommendations. Sports Med 2005;35:71-87.

38. Schmid MR, Hodler J, Cathrein P, Duewell S, Jacob HA, Romero J. Is impingement the cause of jumper's knee? Dynamic and static magnetic resonance imaging of patellar tendinitis in an open-configuration system. Am J Sports Med 2002;30:388-95.

39. Fung DT, Wang VM, Laudier DM, Shine JH, BastaPljakic J, Jepsen KJ, Schaffler MB, Flatow EL. Subrupture tendon fatigue damage. J Orthop Res 2009;27:264-73.

40. Birch HL, Bailey AJ, Goodship AE. Macroscopic "degeneration" of equine superficial digital flexor tendon is accompanied by a change in extracellular matrix composition. Equine Vet J 1998;30:534-9.

41. Smith RK, Birch HL, Goodman S, Heinegård D, Goodship AE. The influence of ageing and exercise on tendon growth and degeneration--hypotheses for the initiation and prevention of strain-induced tendinopathies. Comp Biochem Physiol A Mol Integr Physiol 2002;133:1039-50. 
42. Williams IF, McCullagh KG, Goodship AE, Silver IA. Studies on the pathogenesis of equine tendonitis following collagenase injury. Res Vet Sci 1984;36:326-38.

43. Du J, Bydder M, Takahashi AM, Carl M, Chung CB, Bydder GM. Short T2 contrast with three-dimensional ultrashort echo time imaging. Magn Reson Imaging 2011;29:470-82.

44. Juras V, Apprich S, Szomolanyi P, Bieri O, Deligianni $\mathrm{X}$, Trattnig S. Bi-exponential T2*analysis of healthy and diseased Achilles tendons: an in vivo preliminary magnetic resonance study and correlation with clinical score. Eur Radiol 2013;23:2814-22.

45. Chevrier A, Nelea M, Hurtig MB, Hoemann CD, Buschmann MD. Meniscus structure in human, sheep, and rabbit for animal models of meniscus repair. J Orthop Res 2009;27:1197-203.

46. Cook JL, Feller JA, Bonar SF, Khan KM. Abnormal tenocyte morphology is more prevalent than collagen disruption in asymptomatic athletes' patellar tendons. J Orthop Res 2004;22:334-8.

47. Biskup J, Freeman A, Camisa W, Innes J, Conzemius M. Mechanical properties of canine patella-ligament-tibia segment. Vet Surg 2014;43:136-41.

48. Walters SJ. Consultants' forum: should post hoc sample size calculations be done? Pharm Stat 2009;8:163-9.

49. Juras V, Winhofer Y, Szomolanyi P, Vosshenrich J,

Cite this article as: Pownder SL, Hayashi K, Lin BQ, Meyers KN, Caserto BG, Breighner RE, Potter HG, Koff MF. Differences in the magnetic resonance imaging parameter $\mathrm{T} 2{ }^{*}$ may be identified during the course of canine patellar tendon healing: a pilot study. Quant Imaging Med Surg 2021;11(4):1234-1246. doi: 10.21037/qims-20-684
Hager B, Wolf P, Weber M, Luger A, Trattnig S.

Multiparametric MR imaging depicts glycosaminoglycan change in the Achilles tendon during ciprofloxacin administration in healthy men: initial observation. Radiology 2015;275:763-71.

50. Kijowski R, Wilson JJ, Liu F. Bicomponent ultrashort echo time $\mathrm{T} 2{ }^{*}$ analysis for assessment of patients with patellar tendinopathy. J Magn Reson Imaging 2017;46:1441-7.

51. Diaz E, Chung CB, Bae WC, Statum S, Znamirowski R, Bydder GM, Du J. Ultrashort echo time spectroscopic imaging (UTESI): an efficient method for quantifying bound and free water. NMR Biomed 2012;25:161-8.

52. Liu F, Kijowski R. Assessment of different fitting methods for in-vivo bi-component T2* Analysis of human patellar tendon in magnetic resonance imaging. Muscles Ligaments Tendons J 2017;7:163-72.

53. Johnson DP, Wakeley CJ, Watt I. Magnetic resonance imaging of patellar tendonitis. J Bone Joint Surg Br 1996;78:452-7.

54. Peace KAL, Lee JC, Healy J. Imaging the infrapatellar tendon in the elite athlete. Clin Radiol 2006;61:570-8.

55. Agergaard AS, Malmgaard-Clausen NM, Svensson RB, Nybing JD, Boesen M, Kjaer M, Magnusson SP, Hansen P. UTE T2* mapping of tendinopathic patellar tendons: an MRI reproducibility study. Acta Radiol 2020. [Epub ahead of print]. doi: 10.1177/0284185120918807. 